STUDY OF EFFECT OF THE VARIETY AND ECOLOGICAL FACTERSON AND DISTRIBUTION OF THE DEFERENT LIFE STAGES OF OLIVE PSSYLLA (EUPHYLLURA OLIVINE COSTA ) IN QUNIETRA-KAN AARNBA

Sperij, M. ${ }^{1}$; L. Aslan ${ }^{2}$ and W. Qasis ${ }^{3}$

${ }^{1}$-Qunietra countryside center for (S.A.R)

2. Plant protection Department- Faculty of Agriculture. Damascus Univ.

3 - Plant protection Department- Faculty of Agriculture. Damascus Univ.

دراسة تأثير الصنف النباتي والعوامل المناخية في توزع وانتشـار الأطوار المختلفة

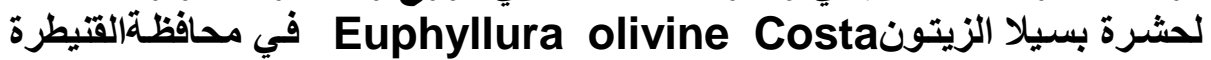

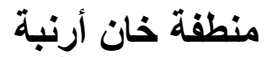

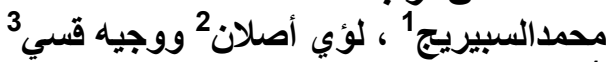
1 1 مركز بحوث القتيطرة

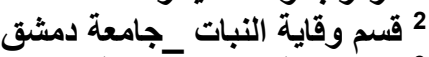

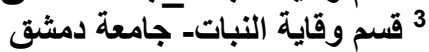

الملخص

تمت دراسـة توزع و انتشـار أطوار حشرة بسيلا الزيتون Euphyllura olivine Costa في

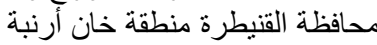

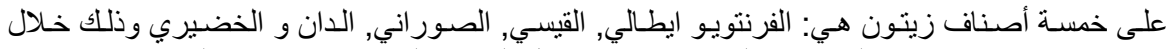

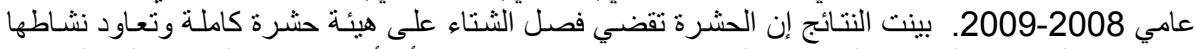

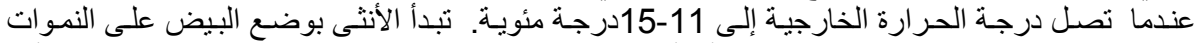

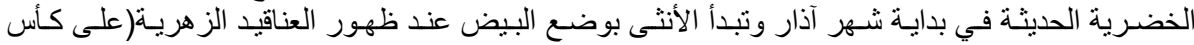

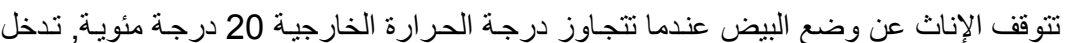

الزهرة).

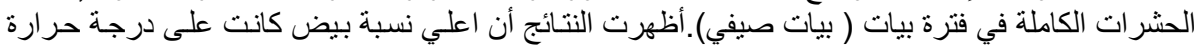

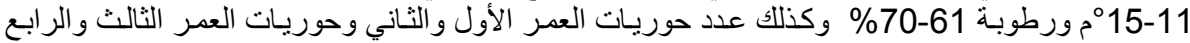

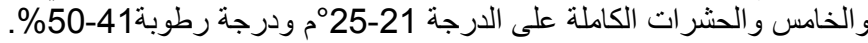

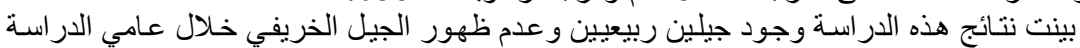

بعكس ماتذكر المر اجع.

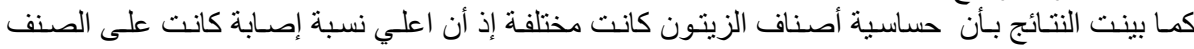

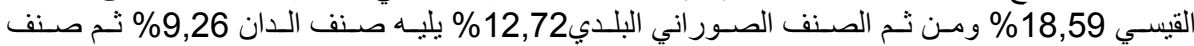

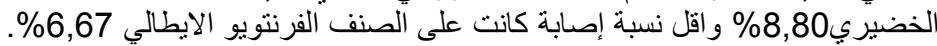

الكلمات المفتاحية :بسيلا الزيتونة عuphyllura olivine Costa. الزيتون, سوريا, القنيطرة

المقدمة

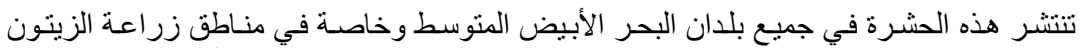

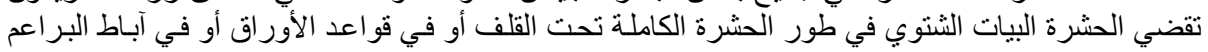

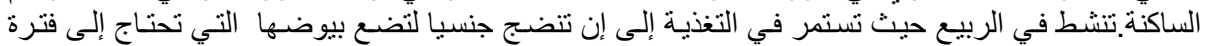

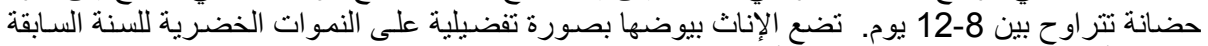

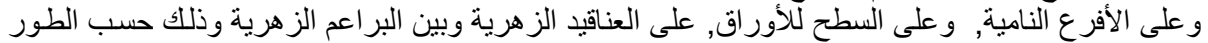

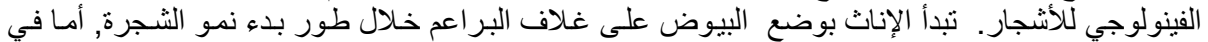

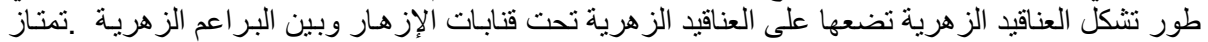




\section{Sperij, M. et al.}

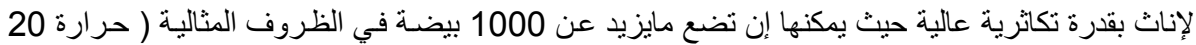

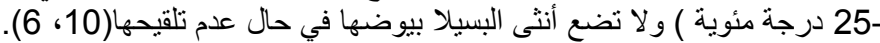

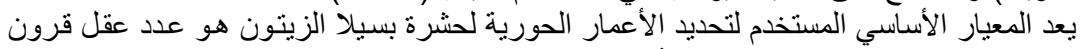

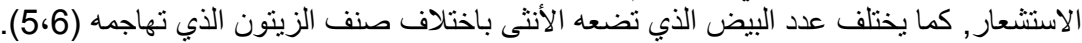

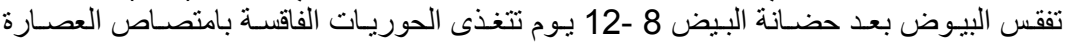

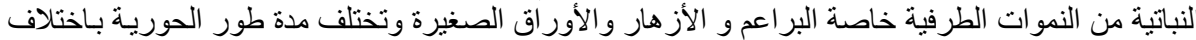

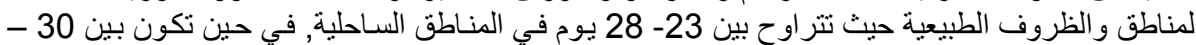

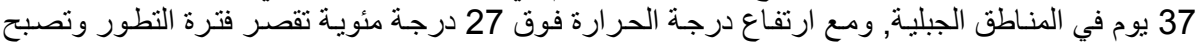

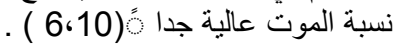

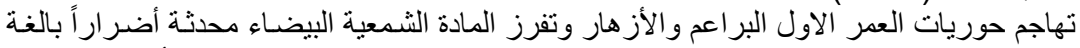

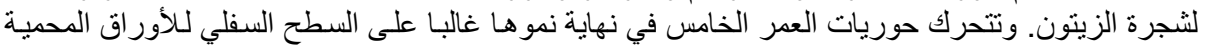

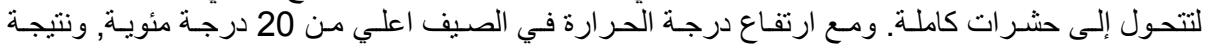

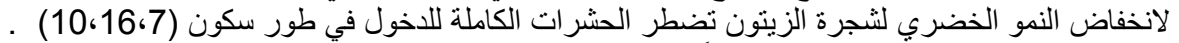

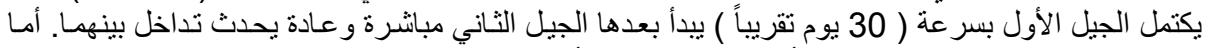

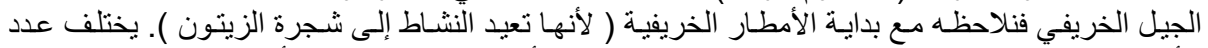

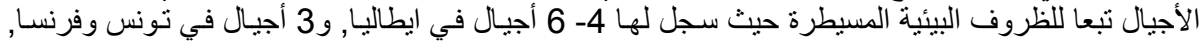

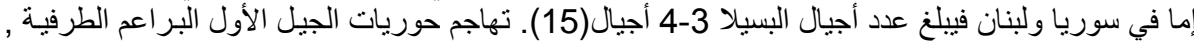

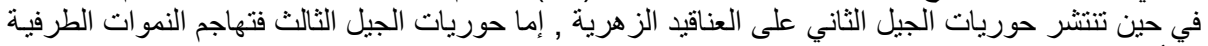

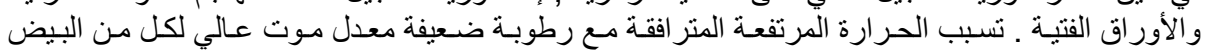

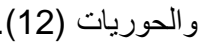

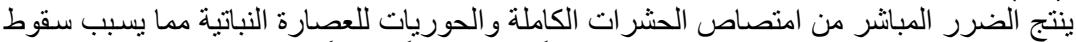

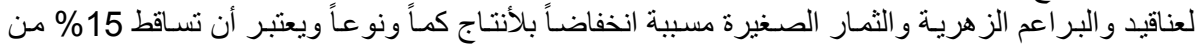

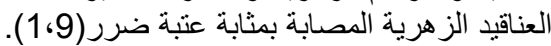

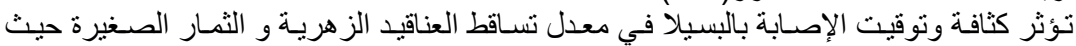

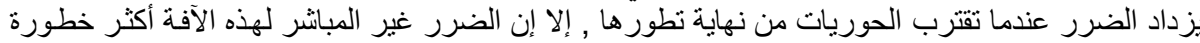

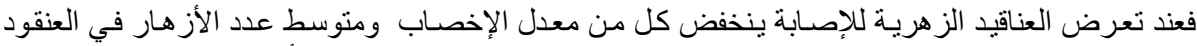

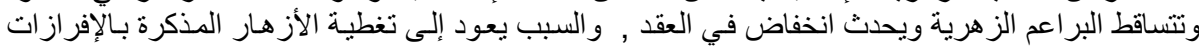

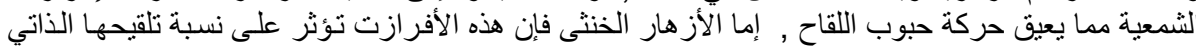

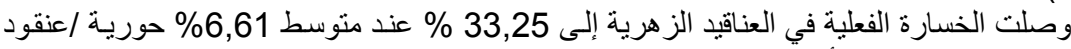

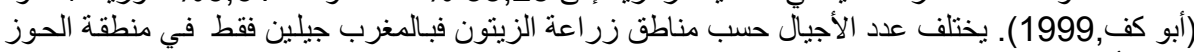

وثلاثة أجبال بمنطقة الصويرة(15) ).

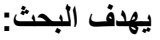
1) دراستة كثافة وتوزع الأطوار و الأعمار المختلفة لحشرة بسيلا الزيتون وارتباطها مع درجات الحرارة و الرطوبة. 2) - دراسة حساسية أصناف الزيتون للإصابة بحشرة بسيلا الزيتون. مواد وطرائق البحث

نفذ البحث خلال عام 2008 و 2009

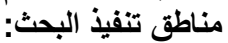

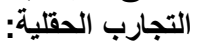

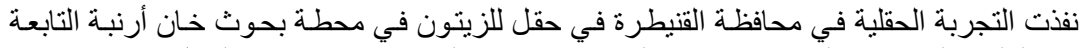

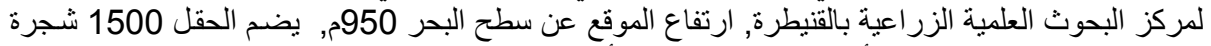

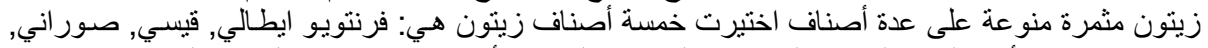

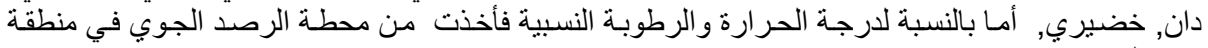
تنفيذ البحث: دان. الاراسات المخبرية وأخذ القراءات: نفذت في مركز بحوث ودر اسات المكافحة الحيوية - كلية الزراعة - جامعة دمثق 

صممت التجربة بطريقة القطاعات العشو ائية الكاملة بواقع خمسة أصناف مختلفة(قطاعات)وبمعدل

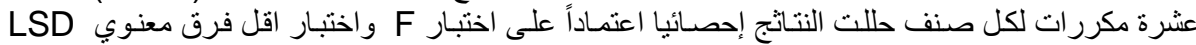

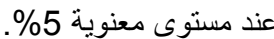

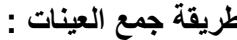
حقليا:

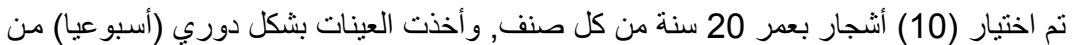

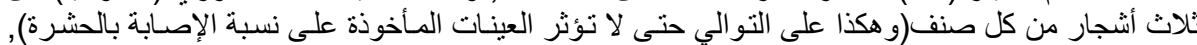

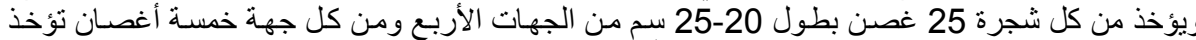

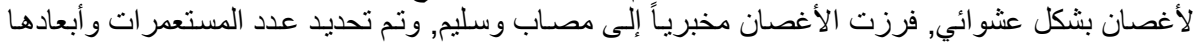

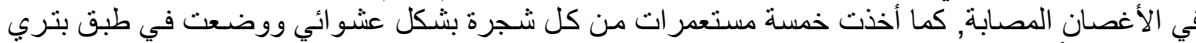

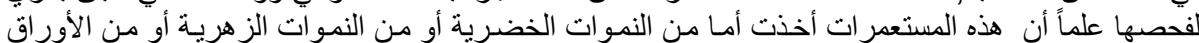
ورذلكة حسب الطور الفينولوجي لثجرة الزيتون.

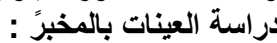

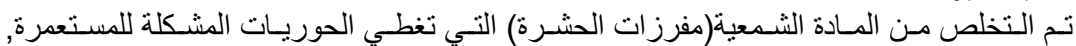

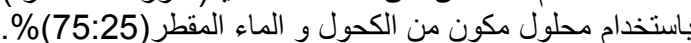

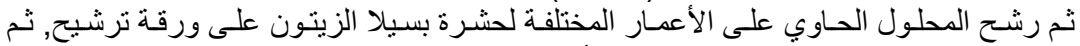

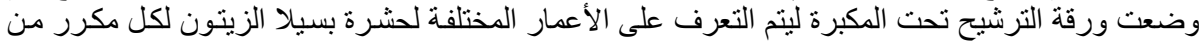
مكررات الأصناف المختلفة.

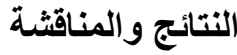

توزيع أطوار بسيلا الزيتون على أصناف الزيتون الخمسة في منطقة خان أرنبة:

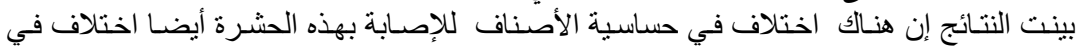

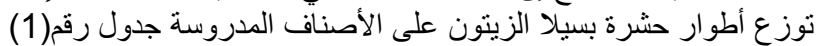

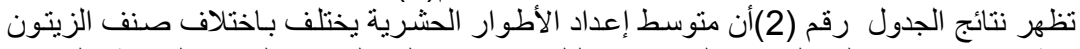

إن أعلى نسبة بيوض كانت على الصنف الصون الصور اني البلدي)

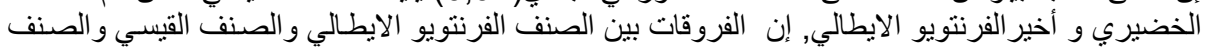

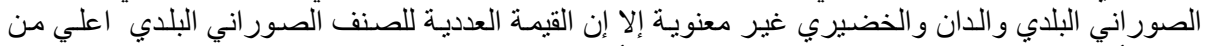

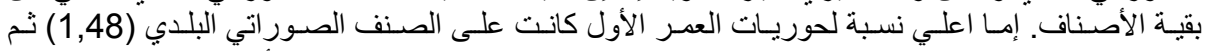

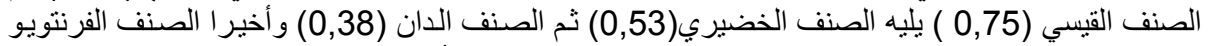

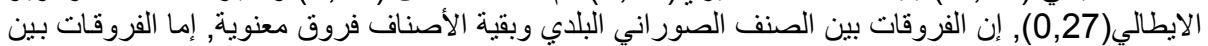

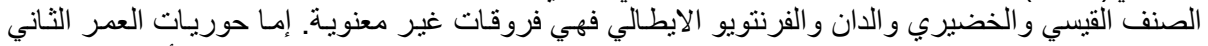

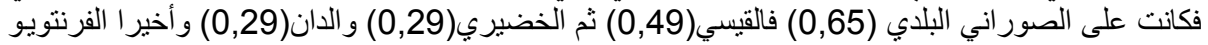

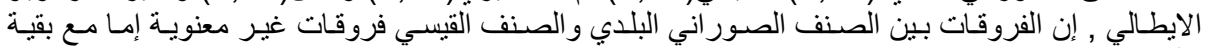

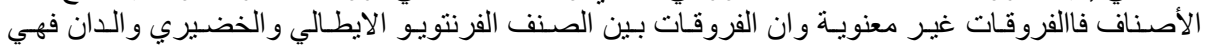

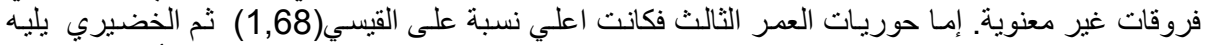

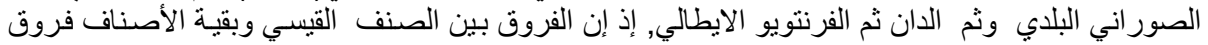

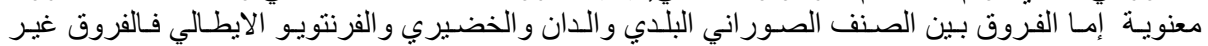

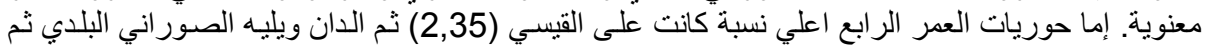

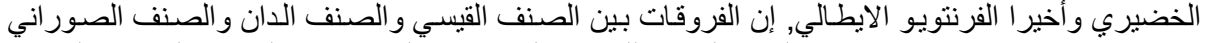

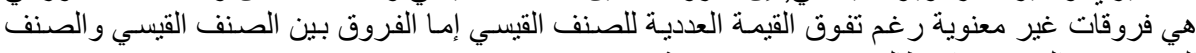

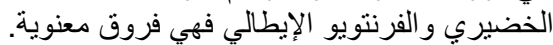




\section{Sperij, M. et al.}




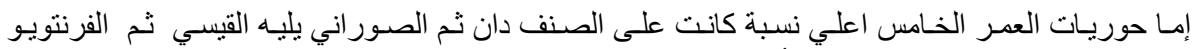

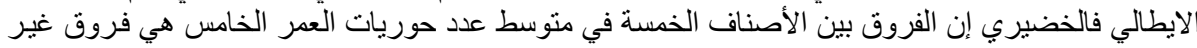

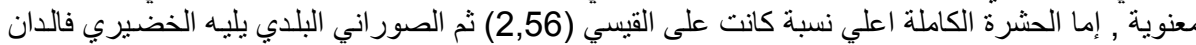

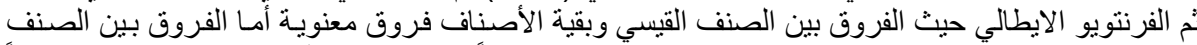

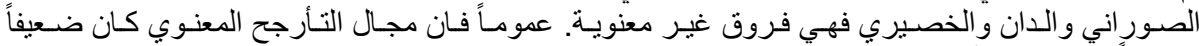
وضئبلاً بين جميع الأصناف وقد غلبت عليها الفروق غير المعنوية باستثناء الحشرات الكاملة فكانت معنوية الكية كما ذكرنا سابقاً.

أظهرت الأصناف المدروسة تباينا واضحا في حساسيتها للإصابة بحشرة بسيلا الزيتون:

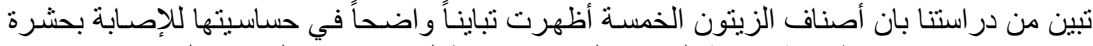

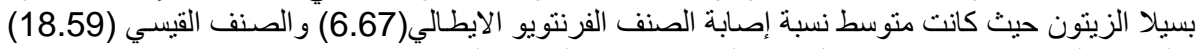
والصنف الصور اني (12.72) والصنف الدان(9.26) والصنف الخضري (8.80) (11).

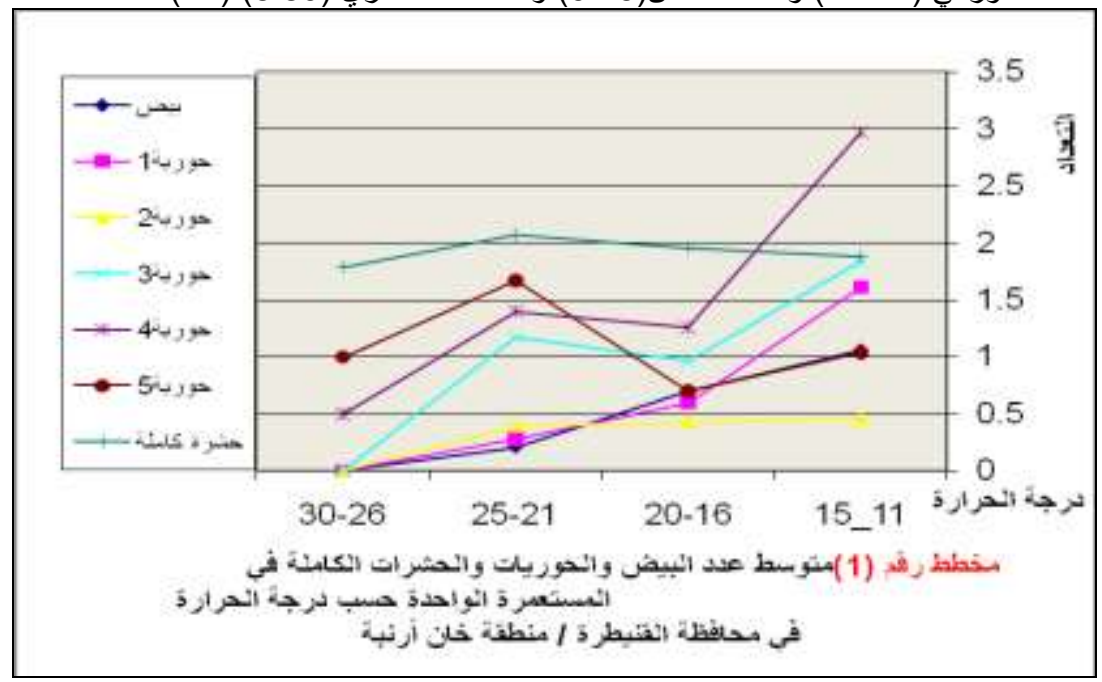

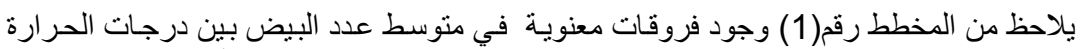

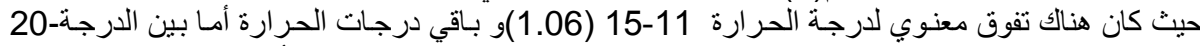

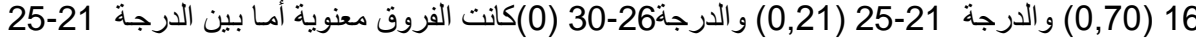
إن

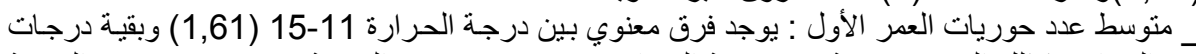
الحر ارة, كذلك الفروق معنوية بين درجة الحر ارة

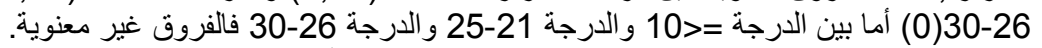

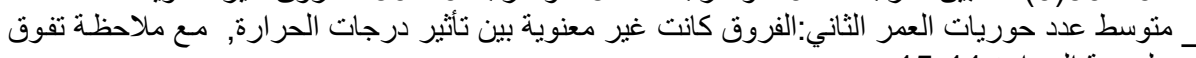

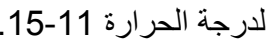

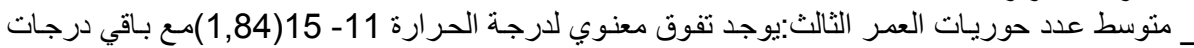

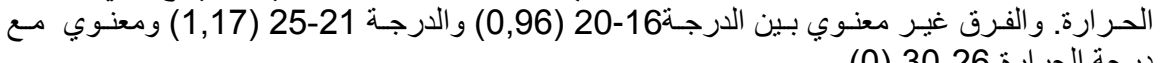

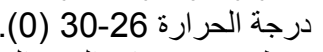

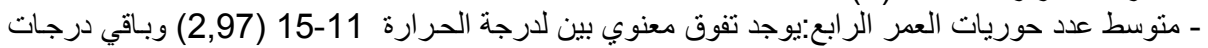

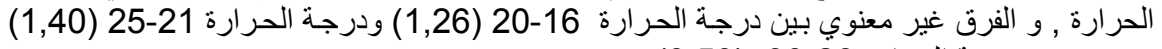

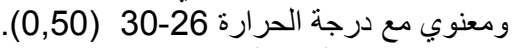

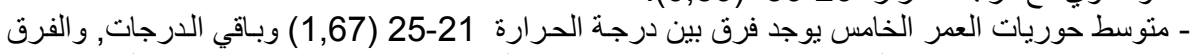

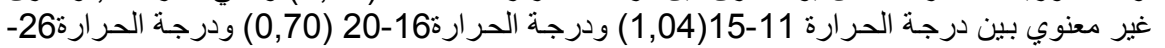




\section{Sperij, M. et al.}

ـ ـ متوسط عدد الحشرة الكاملة : الفروق كانت غير معنويـة بين تأثئير درجات الحرارة, مع تفوق لدرجـة الحرارة 21-25 (2,07) تأثير الرطوبة النسبية في توزع الأطوار المختلفة لحشرة بسيلا الزيتون:

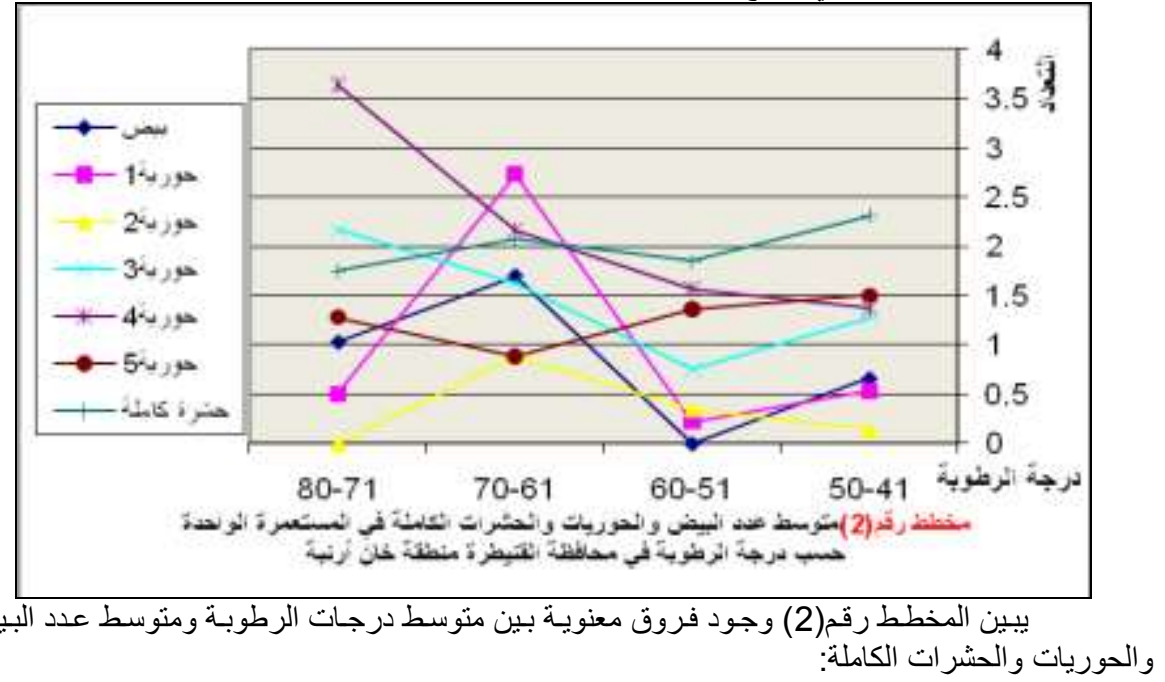

- متوسط عدد البيض يوجد فرق معنوي بن الدرجة الرطوبة 61,70-70 (1,70) وباقي درجات الرطوبة, كنللك

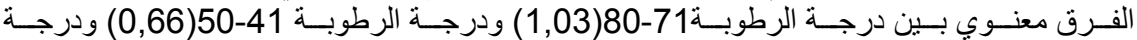

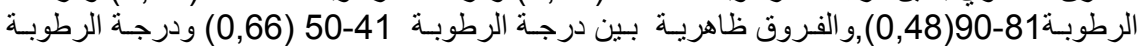
. (0,48)90-81

- متوسط عدد حوريات العمر الأول يوجد فرق معنوي بين درجة الرطوبـ6181-61-70 (2,73) وباقي الدرجات,

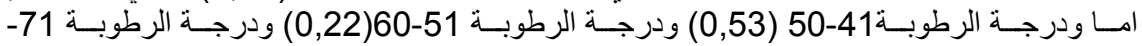

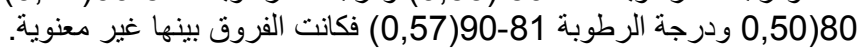

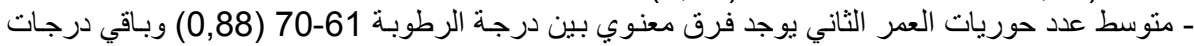

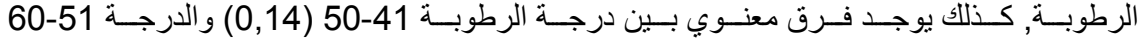

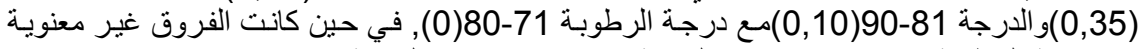

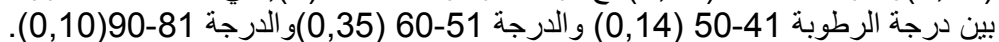

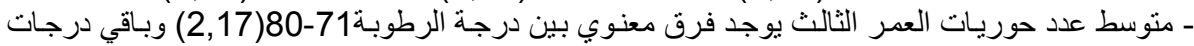

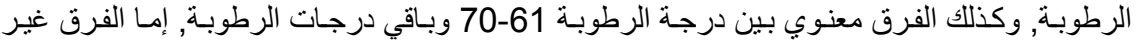

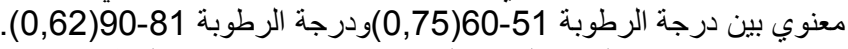

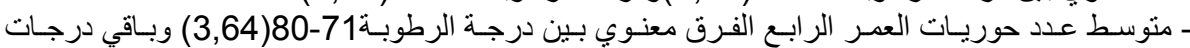

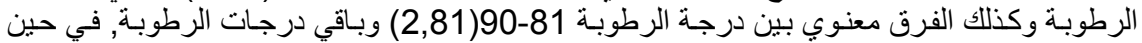

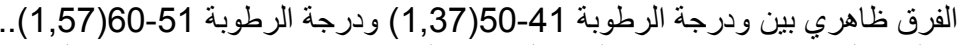

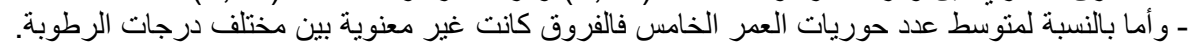

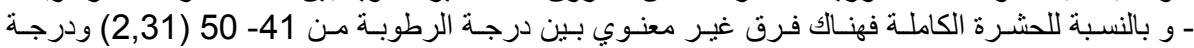

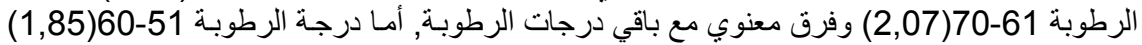
ودرجة الرطوبة 71-80-71) تاثير التفاعل بين درجة الحرارة والرطوبة النسبية في نسبة الإصابة \%

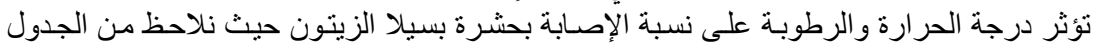

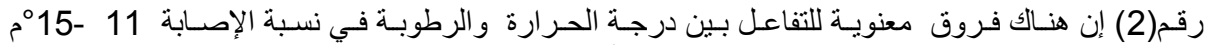

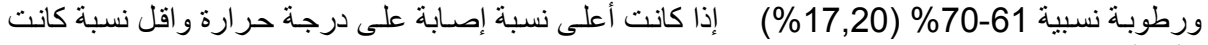

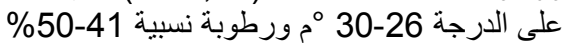
جدول (2): تأثير التفاعل بين درجة الحرارة و الرطوبة النسبية في نسبة الإصـابة (\%) في منطقة خـان أرنبة. 
J. Plant Prot. and Path., Mansoura Univ., Vol. 2 (8), August, 2011

\begin{tabular}{|c|c|c|c|c|c|c|}
\hline \multirow{2}{*}{ معنوية التفاعل } & \multirow{2}{*}{$F_{p}$} & \multicolumn{4}{|c|}{ درجة الرطوبة النسبية \% } & البيان \\
\hline & & $80-71$ & $70-61$ & $60-51$ & $50-41$ & درجة الحرارة \\
\hline \multirow{4}{*}{ *** } & \multirow{4}{*}{8.53} & 11.47 & 17.20 & 0 & 9.07 & $15-11$ \\
\hline & & 0 & 0 & 13.60 & 5.07 & $20-16$ \\
\hline & & 0 & 0 & 11.80 & 12.84 & $25-21$ \\
\hline & & 0 & 0 & 0 & 0 & $30-26$ \\
\hline
\end{tabular}

$$
\begin{aligned}
& \text { 1- من خـلال القراءات التي تم تسجيلها تم ملاحظة جلين للحشرة وههـا الجيلان الربيعيان ولم يلاحظ جيل }
\end{aligned}
$$

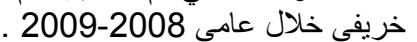

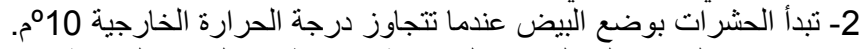

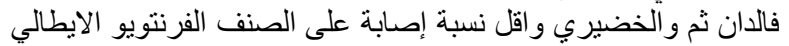

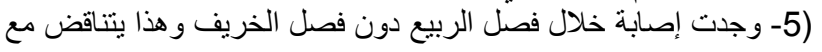

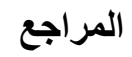

4- Arambourgy,1986. olive tree entomology. UNDP\&FAO.

5- Arambourg Y,1984a. Chermiti B. Euphyllura olivina Costa-Psyllidae. Traité d'entomologie oléicole. Espagne : Conseil oléicole international, 163171

6- Arambourg Y,1984b. Traité d'entomologie oléicole. Espagne : Conseil oléicole international, $360 \mathrm{p}$.

7- Chermiti B.1983. Contribution à l'étude bioécologique du psylle de l'olivier Euphyllura olivina Costa (Hom ; Psyllidae) et de son endoparasite Psyllaephagus euphyllurae Silv. (Hym. Encrytidae). Thèse Doctorat. Ingénieur, Université d'Aix-Marseille, France, $134 \mathrm{p}$.

8- Chermiti, B., 1992. An Approach to the Assessment of the Harmfulness of the Olive Psyllid Euphyllura olivina (Costa) (Homoptera, Aphalaridae). Olivae, 43; 34-42.

9- Froggatt, WW 1901. Notes on selected Australian Hemiptera (Plant bugs). Agricultural Gazette of New South Wales 12 :(1) 592-601.

10- Jervis, M.A., Kidd, N.A.C., 1986. Host-feeding strategies in hymenopteran parasitoids. Biol. Rev. 61, 395-434.

11-. Jarraya A,1986. Bioécologie du psylle de l'olivier, Euphyllura olivina Costa (Hom, Psyllidae) dans la région de Sfax. Sfax, Tunisie : Cinquième session, $20 \mathrm{p}$.

12- Moore, K.M., 1961. Observations on some Australian forest insects. 8. The biology and occurrence of Glycaspis baileyi Moore in New South Wales. Proc. Linnean Soc. New South Wales. 86, 185-200.

13- Patil, N.G., Baker, P.S., Pollard, G.V., 1993. Life histories of Psyllaephagus yaseeni (Hym., Encyrtidae) and Tamarixia leucaenae 
(Hym., Eulophidae), parasitoids of the leucaena psyllid Heteropsylla cubana. Entomophaga 38, 565-577.

14- Prophetou-Athanasiadou DA.1993. Diapause termination and phenology of the olive psyllid, Euphyllura phillyreae on two host plants in coastal northern Greece. Entomol Exp Appl 67 : 193-197.

15- Tajnari H.1992. Étude bio-écologique d'Euphyllura olivina Costa (Hom. Psyllidae) dans les régions du Haouz et d'Essaouira : mise en évidence d'un état de diapause ovarienne. Meknès, Maroc : Thèse de troisième cycle, École nationale d'agriculture, $153 \mathrm{p}$.

16- Yen, A.L., 2002. Short-range endemism and Australian Psylloidea (Insecta: Hemiptera) in the genera Glycaspis and Acizzia (Psyllidae). Invert. Syst. 16, 631-636

STUDY OF EFFECT OF THE VARIETY AND ECOLOGICAL FACTERSON AND DISTRIBUTION OF THE DEFERENT LIFE STAGES OF OLIVE PSSYLLA (EUPHYLLURA OLIVINE COSTA ) IN QUNIETRA-KAN AARNBA

Sperij,M. ${ }^{1}$; L. Aslan ${ }^{2}$ and W. Qasis ${ }^{3}$

${ }^{1}$-Qunietra countryside center for (S.A.R)

2 - Plant protection Department- Faculty of Agriculture. Damascus Univ.

${ }^{3}$ - Plant protection Department- Faculty of Agriculture. Damascus Univ.

\section{ABSTRACT}

The spread and distribution of Euphyllura olivine stages in Daraa were studied on five olive varieties: Frountoyo italic, Qaysi , Sorani, Dan , and Khudyri during the 2008-2009 seasons.

The obtained Results showed that the insect overwintering as adult, which resume its activity when the temperature raised up to $15-11^{\circ} \mathrm{C}$, after that the insect started to lay eggs on the new twigs at the early March with the appearance of flowers as the insect put their eggs at the base of flower.

When the temperature arise above $20^{\circ} \mathrm{C}$, the females stop laying eggs and adult enter into aestivation state

The highest level of egg laying and the individuals of first nymphal instar and adult were recorded at $15-11^{\circ} \mathrm{C}$ and $\mathrm{RH} 61-70 \%$,on the other hand and the third and fourth and fifth nymphal instar and the fifth instar at $21-25^{\circ} \mathrm{C}$ and $\mathrm{RH}, 41-50 \%$.

Two generation were recorded in this investigation as they appear in Spring time, While the Autumn generation did not appear and that was in contrary with the literatures.

The susceptibility of olive varieties varied among olive varieties with the highest level of infection with variety (qaysi) $18,59 \%$, then sorani12,72\%, dan $9,26 \%$, kudayri $8,80 \%$, and the lowest infestation with the variety (frontoyo italic)7,67\% .

Keywords: Olive pssylla, Olive, Syria, Qunietra.

كلية الزراعة - جامعة المنصورة

كلية الزراعة - جامعة المنصورة جاعة المنصورة

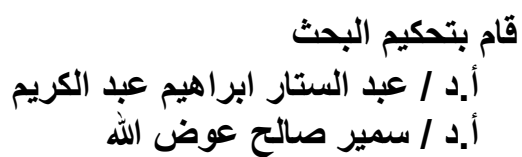


J. Plant Prot. and Path., Mansoura Univ., Vol. 2 (8), August, 2011 
J. Plant Prot. and Path., Mansoura Univ., Vol. 2 (8): 765 - 772, 2011

جدول رقم (1):توزيع أطوار بسيلا الزيتون على أصناف الزيتون الخمسة في منطقة خان أرنبة/ القتيطرة خلال عامي 2008-2009 دراسة تأثير متوسط درجة الحرارة في توزع الأطوار المختلفة لحشرة بسيلا الزيتون:

\begin{tabular}{|c|c|c|c|c|c|c|c|c|c|c|c|c|c|c|c|}
\hline \multicolumn{14}{|c|}{ اطوار الحشرة } & \multirow{3}{*}{$\mathbf{n}$} & \multirow{3}{*}{ البيان } \\
\hline \multicolumn{2}{|c|}{ حشرة كاملة } & \multicolumn{2}{|c|}{ حورية } & \multicolumn{2}{|c|}{ حورية4 } & \multicolumn{2}{|c|}{ حورية 3} & \multicolumn{2}{|c|}{ حورية 2} & \multicolumn{2}{|c|}{ حورية1 } & \multicolumn{2}{|c|}{ بيض } & & \\
\hline SD & $\mathbf{X}$ & SD & $\mathbf{x}$ & SD & $\mathbf{x}$ & SD & $\mathbf{X}$ & SD & $\mathbf{X}$ & SD & $\mathbf{x}$ & SD & $\mathbf{x}$ & & \\
\hline 0.86 & $1.42 \mathrm{c}$ & 1.30 & $0.94 a$ & 1.06 & $1.27 \mathrm{~b}$ & 1.33 & $0.98 \mathrm{~b}$ & 0.38 & $0.17 \mathrm{c}$ & 0.54 & $0.27 \mathrm{~b}$ & 0.65 & $0.29 a$ & 66 & أفرنتويـ \\
\hline 1.22 & $2.56 \mathrm{a}$ & 2.03 & $1.38 \mathrm{a}$ & 2.59 & $2.35 a$ & 1.82 & $1.68 \mathrm{a}$ & 0.93 & $0.49 \mathrm{ab}$ & 1.48 & $0.75 b$ & 1.24 & $0.69 a$ & 88 & لقينيسي \\
\hline 1.02 & $2.01 \mathrm{~b}$ & 1.54 & $1.51 \mathrm{a}$ & 1.88 & 1.79ab & 1.36 & $1.18 \mathrm{~b}$ & 1.18 & $0.65 a$ & 3.70 & $1.48 \mathrm{a}$ & 3.14 & $0.94 a$ & 77 & الزيتون \\
\hline 0.93 & $1.82 \mathrm{~b}$ & 2.63 & $1.62 a$ & 3.39 & $2.00 \mathrm{ab}$ & 1.64 & $1.11 \mathrm{~b}$ & 0.67 & $0.29 b c$ & 1.08 & $0.38 b$ & 1.47 & $0.44 a$ & 66 & إن \\
\hline 1.02 & $1.86 \mathrm{~b}$ & 0.96 & $0.92 a$ & 1.38 & $1.32 \mathrm{~b}$ & 1.24 & $1.21 \mathrm{~b}$ & 0.61 & $0.29 b c$ & 1.14 & $0.53 b$ & 0.85 & $0.43 a$ & 76 & خضبي \\
\hline \multirow{2}{*}{\multicolumn{2}{|c|}{12.41}} & \multicolumn{2}{|c|}{2.36} & \multirow{2}{*}{\multicolumn{2}{|c|}{3.32}} & \multicolumn{2}{|c|}{2.52} & \multicolumn{2}{|c|}{4.00} & \multicolumn{2}{|c|}{4.41} & \multicolumn{2}{|c|}{1.61} & \multicolumn{2}{|r|}{$F_{2}$} \\
\hline & & \multirow{2}{*}{\multicolumn{2}{|c|}{ NS }} & \multirow{2}{*}{\multicolumn{2}{|c|}{0.68}} & \multirow{2}{*}{\multicolumn{2}{|c|}{0.46}} & \multirow{2}{*}{\multicolumn{2}{|c|}{$\frac{* *}{0.25}$}} & \multirow{2}{*}{\multicolumn{2}{|c|}{0.60}} & \multirow{2}{*}{\multicolumn{2}{|c|}{$N S$}} & \multirow{2}{*}{\multicolumn{2}{|c|}{ 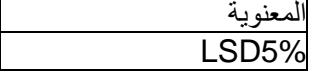 }} \\
\hline \multicolumn{2}{|c|}{0.32} & & & & & & & & & & & & & & \\
\hline
\end{tabular}

